\title{
Novel hemotropic mycoplasmas are widespread and genetically diverse in vampire bats
}

\author{
D. V. VOLOKHOV ${ }^{1} \dagger^{*}$, D. J. BECKER ${ }^{2,3} \uparrow$, L. M. BERGNER ${ }^{4}$, M. S. CAMUS ${ }^{5}$, \\ R. J. ORTON ${ }^{4,6}$, V. E. CHIZHIKOV ${ }^{1}$, S. M. ALTIZER ${ }^{2,3}$ AND D. G. STREICKER ${ }^{2,4,6}$ \\ ${ }^{1}$ Center for Biologics Evaluation \& Research, Food and Drug Administration, Silver Spring, MD, USA \\ ${ }^{2}$ Odum School of Ecology, University of Georgia, Athens, GA, USA \\ ${ }^{3}$ Center for the Ecology of Infectious Disease, University of Georgia, Athens, GA, USA \\ ${ }^{4}$ Institute of Biodiversity, Animal Health and Comparative Medicine, University of Glasgow, UK \\ ${ }^{5}$ Department of Pathology, College of Veterinary Medicine, University of Georgia, Athens, GA, USA \\ ${ }^{6}$ MRC-University of Glasgow Centre for Virus Research, Glasgow, UK
}

Received 9 June 2017; Final revision 11 August 2017; Accepted 17 September 2017;

first published online 24 October 2017

\section{SUMMARY}

Bats (Order: Chiroptera) have been widely studied as reservoir hosts for viruses of concern for human and animal health. However, whether bats are equally competent hosts of non-viral pathogens such as bacteria remains an important open question. Here, we surveyed blood and saliva samples of vampire bats from Peru and Belize for hemotropic Mycoplasma spp.

(hemoplasmas), bacteria that can cause inapparent infection or anemia in hosts. 16S rRNA gene amplification of blood showed 67\% (150/223) of common vampire bats (Desmodus rotundus) were infected by hemoplasmas. Sequencing of the 16S rRNA gene amplicons revealed three novel genotypes that were phylogenetically related but not identical to hemoplasmas described from other (non-vampire) bat species, rodents, humans, and non-human primates. Hemoplasma prevalence in vampire bats was highest in non-reproductive and young individuals, did not differ by country, and was relatively stable over time (i.e., endemic). Metagenomics from pooled D. rotundus saliva from Peru detected non-hemotropic Mycoplasma species and hemoplasma genotypes phylogenetically similar to those identified in blood, providing indirect evidence for potential direct transmission of hemoplasmas through biting or social contacts. This study demonstrates vampire bats host several novel hemoplasmas and sheds light on risk factors for infection and basic transmission routes. Given the high frequency of direct contacts that arise when vampire bats feed on humans, domestic animals, and wildlife, the potential of these bacteria to be transmitted between species should be investigated in future work.

Key words: Chiroptera, Desmodus rotundus, hemoplasmas, metagenomics, phylogenetic analysis, $16 \mathrm{~S}$ rRNA gene, wildlife.

\footnotetext{
* Author for correspondence: D. V. Volokhov, The US Food and Drug Administration, Center for Biologics Evaluation and Research, Laboratory of Method Development, 10903 New Hampshire Avenue, Building 52, Room 1120, Silver Spring, MD 20993-0002, USA.

(Email: dmitriy.volokhov@fda.hhs.gov)

$\dagger$ Both authors contributed equally to this work.
}

\section{INTRODUCTION}

Bats (Order: Chiroptera) have been widely studied as reservoir hosts for pathogens of concern for human and animal health [1,2], with particular attention paid to RNA viruses in the Coronaviridae, Filoviridae, Rhabdoviridae, and Paramyxoviridae 
families [3-5]. The association of bats with the human and animal diseases is in part explained by the high diversity of zoonotic viruses that circulate in bats. Per species, bats host more zoonotic viruses than all other mammalian orders and are more likely to share viruses among species, which may be due to aggregation of large colonies, migration, and the multi-species roosts of many bats [6-8]. However, whether bats are equally competent hosts of non-viral pathogens such as bacteria remains an open and understudied question [9,10]. Bacteria such as Yersinia spp. and Leptospira spp. have been detected in bats [11,12], but the importance of these pathogens for human, wildlife, or domestic animal health remains unknown. For other bacteria such as Bartonella spp., phylogenetic analyses have suggested a potential role of bats in the transmission of zoonotic Bartonella sp. [13], such as Bartonella mayotimonensis, an etiologic agent of human endocarditis [14,15].

Recent studies have also shown hemotropic Mycoplasma spp. (hemoplasma) infections in bats [16-19]. Hemoplasmas are facultative intracellular erythrocytic bacteria without a cell wall that were formerly classified as Haemobartonella and Eperythrozoon spp., and were re-classified as Mycoplasma spp. based on their 16S rRNA gene sequences and cell morphologic properties [20-23]. These bacteria are thought to be transmitted through direct (blood and saliva) and possibly vector-borne contact [23-26] and can cause acute and chronic anemia in wildlife, humans, and domestic animals [27-30], particularly in immunocompromised hosts [31,32]. Almost all Mycoplasma spp., including hemoplasmas, appear to show host specificity that seems to be a result of the host-pathogen interaction during evolution; however, potential zoonotic or inter-species transmission has also been reported [30,31,33-35]. Among bat species studied to date, 16S rRNA gene sequence analyses have shown that hemoplasmas identified in little brown bats (Myotis lucifugus) from the USA demonstrated the closest homology $(\sim 92 \%)$ with a hemoplasma detected in a human, Candidatus Mycoplasma haemohominis, and with Mycoplasma haemomuris detected in a small Japanese field mouse (Apodemus argenteus) $[16,36]$. Recent work on Neotropical bat species from Brazil found velvety free-tailed bats (Molossus molossus) were infected with hemoplasmas that shared close identity (93-96\%) with a hemoplasma detected in mice, Mycoplasma coccoides [19]. Surveys of Schreibers' bats (Miniopterus schreibersii) and one long-eared bat (Myotis capaccinii) also detected hemoplasma species with close identity $(97 \%)$ to Candidatus Mycoplasma haemohominis [17]. These phylogenetic relationships between bat hemoplasmas and hemoplasmas from other species suggest possible cross-species transmission in history [37], which may be relevant for zoonotic transmission from bat species with frequent contact with humans.

No published data currently exist on evidence for hemoplasma infection in vampire bats or on the prevalence and diversity of these bacteria in hematophagous bats. Yet owing to their direct contact with mammals through blood feeding, vampire bats are an obvious candidate species for which to assess hemoplasma infection and phylogenetic relationships to genotypes previously described in other mammals, including humans and non-human primates. Three species comprise the subfamily Desmodontinae: the common vampire bat (Desmodus rotundus), the hairy-legged vampire bat (Diphylla ecaudata), and the white-winged vampire bat (Diaemus youngi). Vampire bats occur across diverse habitat types throughout Latin America, ranging from Mexico to northern Argentina [38]. While these species historically feed on wild mammals and birds, the most abundant species, D. rotundus, preferentially feed on livestock and poultry owing to the greater accessibility and reliability of these novel prey species [39-41]. $D$. rotundus also commonly feeds on humans, making it an important source of human rabies virus outbreaks [3,42-44]. As biting is a possible transmission route for hemoplasmas in other mammals through exposure to infectious blood or saliva [24-26], vampire bat feeding behavior could possibly facilitate transmission to humans, domestic animals, and wildlife. Infection in vampire bats roosting in anthropogenic habitats could also enhance vector-borne transmission cycles [23]. The goals of our study were thus: (i) to identify hemoplasma species in vampire bats; (ii) to assess the position of detected sequences within the broader hemoplasma phylogeny; (iii) to identify risk factors for infection, including age and seasonality [45]; and (iv) to determine hemoplasma presence in vampire bat saliva to assess the possibility of direct transmission of these bacteria.

\section{MATERIALS AND METHODS}

\section{Vampire bat sampling}

During 2015 and 2016, we sampled 224 vampire bats across 14 sites in Peru (Departments of Amazonas 
[AM], Apurimac [API], Ayacucho [AYA], Cajamarca [CA], and Loreto [LR]; $n=12$ ) and in Orange Walk [OW] District $(n=2)$, Belize (Fig. 1). We sampled sites 1-2 times annually (Supplementary Table S1), in which bats were captured in mist nets or harp traps placed at exits of roosts, along flight paths, or outside livestock corrals from 19:00 to 05:00 [46,47]. Upon capture, bats were held in individual cloth bags and issued a uniquely coded incoloy wing band ( $3.5 \mathrm{~mm}$, Porzana Inc.). Bats were classified as subadult or adult based on the fusion of phalangeal epiphyses [48], and reproductive activity was indicated by the presence of scrotal testes in males and pregnancy or lactation in females. We obtained blood by lancing the propatagial vein with a sterile 23 -gauge needle, followed by sample collection with heparinized capillary tubes. To screen for hemoplasmas by PCR, up to $30 \mu \mathrm{l}$ blood was stored on Whatman FTA cards to preserve bacterial DNA [49]. The whole blood-impregnated FTA cards were stored in individual pouches at room temperature with desiccant until laboratory analysis. Thin blood smears were prepared on glass slides, stained with buffered Wright-Giemsa (Camco Quik Stain II, Fisher Scientific), and screened by a board-certified veterinary clinical pathologist (MSC) for hemoplasmas using a light microscopic examination of a representative area of the blood monolayer at $1000 \times$ magnification. For assessment of hemoplasmas presence in vampire bat saliva, we collected oral swabs from Peru; samples were preserved in $2 \mathrm{ml}$ of RNAlater (Invitrogen) at $-80^{\circ} \mathrm{C}$ until laboratory analyses.

All field procedures were approved by the University of Georgia Animal Care and Use Committee (A2014 04-016-Y3-A5) and the University of Glasgow School of Medical Veterinary and Life Sciences Research Ethics Committee (Ref08a/15). Bat capture and sampling were authorized by the Belize Forest Department under permits CD/60/3/15(21) and WL/ 1/1/16(17) and by the Peruvian Government under permits RD-009-2015-SERFOR-DGGSPFFS, RD264-2015-SERFOR-DGGSPFFS, and RD-142-2015SERFOR-DGGSPFFS. Access to genetic resources from Peru was granted under permit RD-054-2016SERFOR-DGGSPFFS.

\section{DNA extraction, PCR amplification, and sequencing of amplicons}

Genomic DNA was extracted from 3 to $52 \mathrm{~mm}$ punches of blood preserved on Whatman FTA cards using QIAamp DNA Investigator Kits (Qiagen, Hilden, Germany) following the manufacturer's instructions. DNA samples were stored at $-80^{\circ} \mathrm{C}$ until use.

Primary screening for the presence of hemoplasmas was performed with PCR using previously published UNI_16S_mycF and UNI_16S_mycR universal primers for amplification of the partial 16S rRNA hemoplasma genes [50]. Based on our previously published data [50] and recent in silico analysis [51] using BLAST search (https://blast.ncbi.nlm.nih.gov/Blast. cgi) of these universal primers against the different mycoplasma 16S rRNA gene sequences available in GenBank, these primers produce PCR fragments with size of approximately 1000-1035 bp (depending on the target Mycoplasma spp.). If samples were considered strongly positive for infection with hemoplasmas in the primary PCR screening, we also amplified the full-length 16S rRNA gene (approximately 1450-1560 nt) using PCR primers designed in this study (16SF-AGAGTTTGATCCTGGCT CAG and 16SR-CTCAAAACTGAAAGYCATC CGC) and then sequenced these amplicons (see GenBank accession numbers KY932674, KY932675, KY932677-KY932680, KY932687-KY932693, KY 932695, KY932696, KY932701， KY932703-KY 932710, KY932712, KY932716, KY932721, and KY932722). If samples were weakly positive (i.e., a weak band) in the primary PCR screening, we did not amplify the full-length 16S rRNA gene but instead sequenced amplicons of the partial 16S rRNA gene from the primary PCR (see GenBank accession numbers KY932676, KY932681-KY932686, KY932694, KY932697-KY932700, KY932702, KY932711, KY932713-KY932715，KY932717-KY932720，KY 932723, KY932724). All PCRs in this study were qualitative and thus a load of hemoplasma DNA in individual blood samples was not quantified.

The 16S rRNA amplicons produced were directly sequenced (without cloning into a plasmid vector) by Macrogen (https://www.macrogenusa.com; Rockville, MD, USA). Prior to sequencing, PCR amplicons were purified by electrophoresis through $1 \cdot 5 \%$ agarose gels and extracted with the QIAquick Gel Extraction Kit (Qiagen, Hilden, Germany). Amplicons were sequenced with the same primers used for PCR amplification and then with internal (walking) primers when needed. The amplification mixture for all PCR contained $5 \mu$ l of $10 \times$ HotStarTaq PCR buffer, 1.5 $\mathrm{mM} \mathrm{MgCl}_{2}, 200 \mathrm{mM}$ dNTP mixture, $1 \mathrm{mM}$ of each primer, and $2.5 \mathrm{U}$ HotStarTaq Plus DNA 
(a)

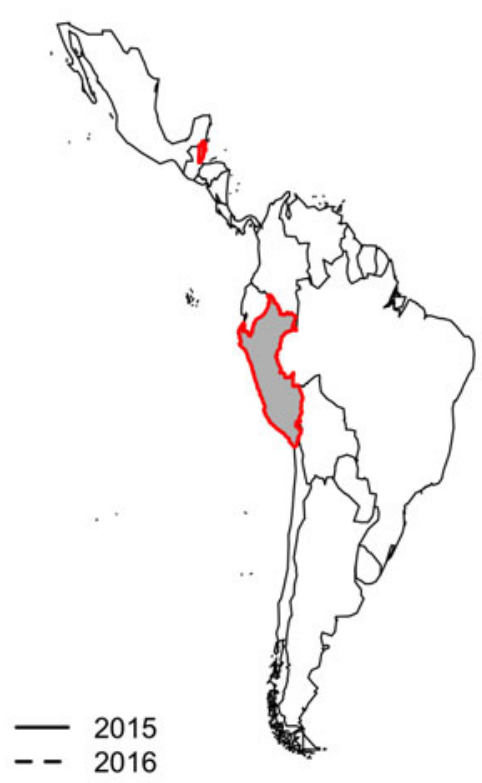

(b) ow1
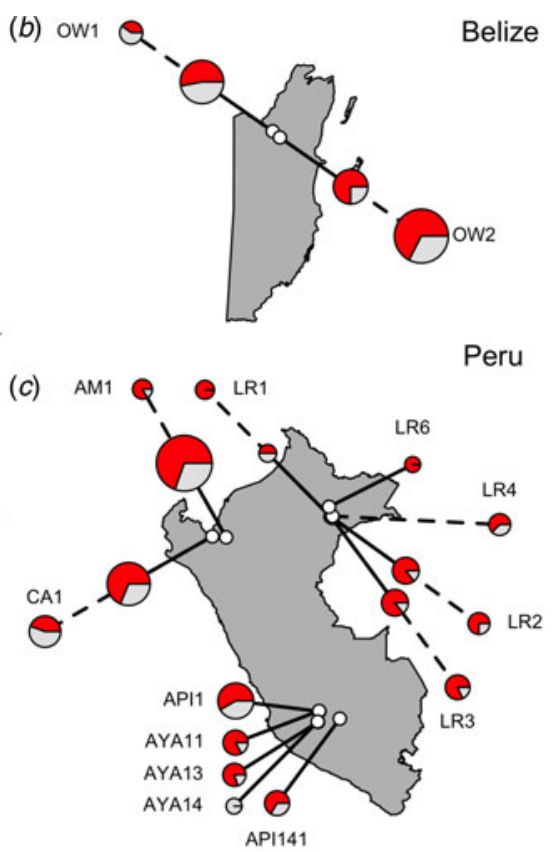

Fig. 1. Location of vampire bat sampling sites in Latin America, with Belize and Peru showed in gray with red outlines (a). Insets show the location of field sites (white) and the prevalence of hemoplasmas per site $(b$ and $c$ ) across study years (solid line $=2015$, dashed line $=2016$ ), with red denoting the proportion of infected bats. Points are scaled by sample size.

Polymerase (Qiagen, Hilden, Germany) in a final volume of $50 \mu \mathrm{l}$, including $3 \mu \mathrm{l}$ of DNA template. The Vent DNA Polymerase Kit (New England Biolabs), which contains high-fidelity thermophilic Vent DNA polymerase, was also used for the amplification of PCR products for subsequent sequencing. The absence of PCR inhibitors in isolated blood DNA was confirmed by PCR amplification of the D. rotundus mitochondrial 16S rRNA gene as an extraction positive control (with primers 16S_Desmodus_FAACAGCAAAGCTTACCCCTTGTACC and 16S Desmodus_R-GTCTGAACTCAGATCACGTAGG AC). Negative (no DNA added) controls were run for each PCR, and Candidatus Mycoplasma haemozalophi [50] was used as a positive control.

All PCR reactions were conducted under the following conditions: a polymerase activation step at $95^{\circ} \mathrm{C}$ for $5 \mathrm{~min}$ (or $15 \mathrm{~min}$ for HotStarTaq only) followed by 45 cycles of $95^{\circ} \mathrm{C}$ for $30 \mathrm{~s}, 60^{\circ} \mathrm{C}$ for $60 \mathrm{~s}$, and $72{ }^{\circ} \mathrm{C}$ for $60 \mathrm{~s}$, with a final extension at $72{ }^{\circ} \mathrm{C}$ for $10 \mathrm{~min}$. PCR products were detected by electrophoresis through 1\% TAE-agarose gels containing ethidium bromide followed by UV visualization.

To avoid the potential presence of chimeric sequences or PCR-derived variants in the data, all hemoplasma 16S rRNA PCR products for phylogenetic analyses were directly amplified from blood DNA samples of vampire bats with two different DNA polymerases (HotStarTaq and Vent) and were directly sequenced without cloning [52,53]. All gene sequences prior to the downstream phylogenetic analysis were subjected to the chimeric sequence analysis using DECIPHER [54] and UCHIME [55]. All sequences available from this study have been deposited in GenBank under the accession numbers KY932674 KY932724.

\section{Phylogenetic analyses}

The 16S rRNA sequences determined in this study were compared to those available in GenBank using procedures, algorithms, and methods for phylogenetic tree inference as described elsewhere [50,56,57]. Briefly, the sequences of the 16S rRNA genes were compared with the GenBank nucleotide database. Nucleotide sequences were aligned using the publicly available Clustal X software (http://www.clustal.org). Inter- and intra-species similarity were generated using BioEdit software (http://www.mbio.ncsu.edu/ BioEdit/bioedit.html). Genetic distances were calculated by using the Kimura two-parameter and Tamura-Nei models, and phylogenetic trees were constructed in MEGA 6 software using the minimum evolution algorithm (http://www.megasoftware.net). 


\section{Statistical analyses}

We first calculated hemoplasma prevalence and 95\% confidence intervals using the Wald method in the prevalence package of $\mathrm{R}$ [58]. We then tested if hemoplasma genotypes detected via sequencing were associated with geography, bat demography, or time using Chi-squared tests with $p$ values generated via a Monte Carlo procedure with 1000 simulations [59]. We used the Benjamini and Hochberg correction to adjust $p$ values for multiple comparisons [60].

We used generalized mixed effects models (GLMMs) with binomial errors and a logit link to determine risk factors for hemoplasma infection status (positive or negative) [61]. Bat ID was included a random effect to account for multiple sampling of a small number of individuals $(n=6)$; the site was not included as a random effect owing to repeatedly failed model convergence. Using a reduced dataset free of missing values $(n=220)$, we compared a set of GLMMs with country, bat age, sex, reproductive status, year, and season (spring, summer, fall) as fixed effects alongside possible two-way interactions; we limited the number of models to roughly $50 \%$ our sample. We compared models with Akaike information criterion corrected for small sample size (AICc) and calculated marginal and conditional $R^{2}$ values to assess fit $[62,63]$. We performed model averaging to compute mean odds ratios (OR) and 95\% confidence intervals across the set of GLMMs whose cumulative Akaike weight $\left(w_{i}\right)$ summed to $95 \%$; mean ORs were standardized with partial standard deviation [64]. We used the MuMIn and lme4 packages for model averaging $[65,66]$.

\section{Assessment of hemoplasmas in saliva}

To examine the possibility for direct transmission of hemoplasmas through biting or grooming, we used metagenomic data from a parallel study to screen vampire bat saliva samples from the same regions of Peru where blood samples were collected. Although a PCR-based screening of saliva samples would have made for the most comparable set of results, untargeted metagenomic sequencing has been found equally or more sensitive for pathogen detection compared with conventional PCR [67-69]. Five saliva pools were shotgun sequenced, each of which contained nucleic acid extractions from saliva swabs often vampire bats from 1 to 2 colonies within each department (Amazonas, Cajarmarca, Loreto,
Ayacucho) or across two neighboring departments (Ayacucho and Apurimac). Pooled samples represent the same regions of Peru, though not necessarily the same colonies or individuals, tested for hemoplasmas in blood through PCR.

Total nucleic acid was extracted from swabs using a modified protocol with the BioSprint 96 OneFor-All Vet Kit (Qiagen, Hilden, Germany) and a KingFisher Flex 96 machine (Thermo Fisher Scientific). Swabs were incubated twice consecutively in tubes containing lysis buffer (Buffer RLT) and Proteinase $\mathrm{K}$ for $15 \mathrm{~min}$ at $56^{\circ} \mathrm{C}$; volume from the two tubes was combined prior to the addition of other extraction reagents, and the manufacturer's protocol was subsequently followed. Extractions were quantified using a Qubit RNA HS Assay Kit (Thermo Fisher Scientific) and pooled at approximately $120 \mathrm{ng}$ RNA per sample. Pools were treated for $5 \mathrm{~min}$ at $35^{\circ} \mathrm{C}$ with $2 \mathrm{U}$ DNase I (Ambion) and cleaned with a $1.8 \times$ ratio of Agencourt RNAClean XP beads (Beckman Coulter). Pools were then depleted for host ribosomal RNA using the RiboZero rRNA Removal Kit (Human/Mouse/Rat) (Illumina) per the manufacturer's instructions. Prior to library preparation, cDNA synthesis was performed using the Maxima $\mathrm{H}$ Minus First Strand cDNA Synthesis Kit (Thermo Fisher Scientific) and the NEBNext mRNA Second Strand Synthesis Module (New England Biolabs). Samples were library prepared using the KAPA DNA Library Preparation Kit for Illumina (KAPA Biosystems), at which point they were individually barcoded with primers designed based on the NEBNext Multiplex Oligos for Illumina Index Primers (New England Biolabs). The libraries included in this study were combined in equimolar ratios with other metagenomic libraries for sequencing on an Illumina NextSeq500 at the University of Glasgow Centre for Virus Research.

Reads were demultiplexed according to barcode and quality filtered using TrimGalore [70,71] with a quality threshold of 25 , minimum read length of 75 $\mathrm{bp}$, and clipping the first $14 \mathrm{bp}$ of the read. Low complexity reads were filtered out using the DUST method and PCR duplicates removed using PRINSEQ [72]. We screened cleaned reads for hemoplasma-like sequences using nucleotide BLAST [73] against a custom database composed of the PCR-generated hemoplasma sequences from this study, retaining only the best alignment for a single query-subject pair. The hemoplasma-like reads were then de-novo assembled using the assembly-only function of SPAdes [74], 
and contigs greater than $300 \mathrm{bp}$ were screened for sequences closely matching Mycoplasma species using nucleotide BLAST in Genbank.

\section{RESULTS}

\section{Hemoplasma genotype detection and phylogenetic analysis}

Hemoplasma infection was detected by $16 \mathrm{~S}$ rRNA PCR in $150 / 223(67 \% ; 95 \% \mathrm{CI}=0 \cdot 61-0 \cdot 73)$ of common vampire bats (D. rotundus) but was not found in our single sample from a hairy-legged vampire bat (Diphylla ecuadata). We did not detect hemoplasmas in any blood samples with light microscopy. Hemoplasma infection prevalence as assessed by PCR ranged from 0 to $100 \%$, with a mean $67.53 \%$ bats per site infected with at least one genotype (Fig. 1).

Figure 2 shows the inferred phylogenetic position of the hemoplasma sequences identified in vampire bats among known hemotropic Mycoplasma species using partial sequences of the 16S rRNA genes (871-890 bp). Vampire bat hemoplasmas represented three main genotypes (Supplementary Table S2, Fig. 2). One other sample (D141; GenBank accession number KY932724) showed $97 \%$ similarity to the 16 S ribosomal RNA gene of Mycoplasma moatsii strain MK405 (NR_ 025186), a non-hemotropic Mycoplasma spp. isolated from grivet monkeys (Cercopithecus aethiops); however, we were not able to amplify the full-length $16 \mathrm{~S}$ rRNA gene from this sample. Inter-laboratory contamination with $M$. moatsii was excluded as we do not handle this species in our laboratory; the same sequence was also repeatedly amplified from the same blood sample. Vampire bat hemoplasma genotypes 1 and 2 were closely related (97-98\% intergenotype similarity, Supplementary Table S2) and similar to hemoplasmas detected in common bentwing bats (M. schreibersii) in Spain (86-87\% similarity to GenBank accession numbers KM538691KM538698), little brown bats (Myotus lucifugus) in the USA (88-89\% similarity to KF713538), wild Japanese monkeys (Macaca fuscata) (93-94\% similarity to AB820288), tufted capuchins (Sapajus apella) in the Brazilian Amazon (88-90\% similarity to KT314160-KT314164), and to a hemoplasma detected in a human patient with hemolytic anemia and pyrexia in the USA $(94 \%$ similarity to GU562823). Genotype 3 was most similar to M. coccoides (93-95\% similarity to AY171918),
Candidatus Mycoplasma turicensis (93-94\% similarity to DQ157153), a hemoplasma detected in a capybara (Hydrochoerus hydrochaeris) in Brazil (92-93\% similarity to FJ667774), and hemoplasmas detected in velvety free-tailed bats (M. molossus) in Brazil (90-93\% similarity to KY356747-KY356751). No chimeras were detected from these 16S rRNA gene sequences.

Within vampire bats, hemoplasma genotype 1 was the most common sequence identified (Fig. 3), infecting $68 \%$ of positive bats (100/150). Genotypes 2 and 3 infected $22 \%(33 / 150)$ and $9 \%(14 / 150)$ of positive bats, respectively. The number of genotypes detected per site ranged from one to three, but no coinfection with multiple genotypes was observed. Genotype 1 was detected across all sites, genotype 2 was detected in both Belize sites and $40 \%$ of Peru sites (primarily northern and eastern Amazon), genotype 3 was detected in both Belize sites and $40 \%$ of Peru sites. The M. moatsii-like hemoplasma sequence was detected in only one bat in Belize. The three novel genotypes showed no association with country $\left(\chi^{2}=3 \cdot 09\right.$, $P=0.31)$, site $\left(\chi^{2}=30 \cdot 14, P=0.31\right)$, department $\left(\chi^{2}=\right.$ $13 \cdot 68, P=0 \cdot 31)$, age $\left(\chi^{2}=0 \cdot 28, P=0 \cdot 88\right)$, season $\left(\chi^{2}=\right.$ $8 \cdot 65, P=0 \cdot 21)$, or year $\left(\chi^{2}=1 \cdot 48, P=0.59\right)$ but were associated with reproduction $\left(\chi^{2}=11.32, P=0.03\right)$ and were marginally associated with $\operatorname{sex}\left(\chi^{2}=7 \cdot 12\right.$, $P=0 \cdot 10)$. Non-reproductive bats showed greater infection with genotype 1 but less infection with genotype 2 than reproductive bats, while males tended to harbor more infection with genotypes 1 and 2 than females (Supplementary Fig. S1).

\section{Risk factors for hemoplasma infection in vampire bats}

All hemoplasma genotypes and vampire bat species were pooled for analyses of infection prevalence. The 95\% confidence set of GLMMs contained $58 / 124$ of the original models (Supplementary Table S3), with variable importance as follows: reproductive status $(95 \%)$, age $(75 \%)$, season $(65 \%)$, the interaction between season and reproductive status $(48 \%)$, sex $(44 \%)$, country $(27 \%)$, year $(18 \%)$, the interaction between season and sex $(7 \%)$, the interaction between sex and reproductive status (7\%), and the interaction between country and year $(<1 \%)$. These models explained between $9 \%$ and $21 \%$ of the variation in hemoplasma infection (Supplementary Table S3). The average odds ratios for the effect of being non-reproductive $(\mathrm{OR}=1.45,95 \% \mathrm{CI}=1 \cdot 05$ $2.02)$ and being non-reproductive in the spring (OR $=0 \cdot 68,95 \% \mathrm{CI}=0 \cdot 50-092)$ on infection was different 


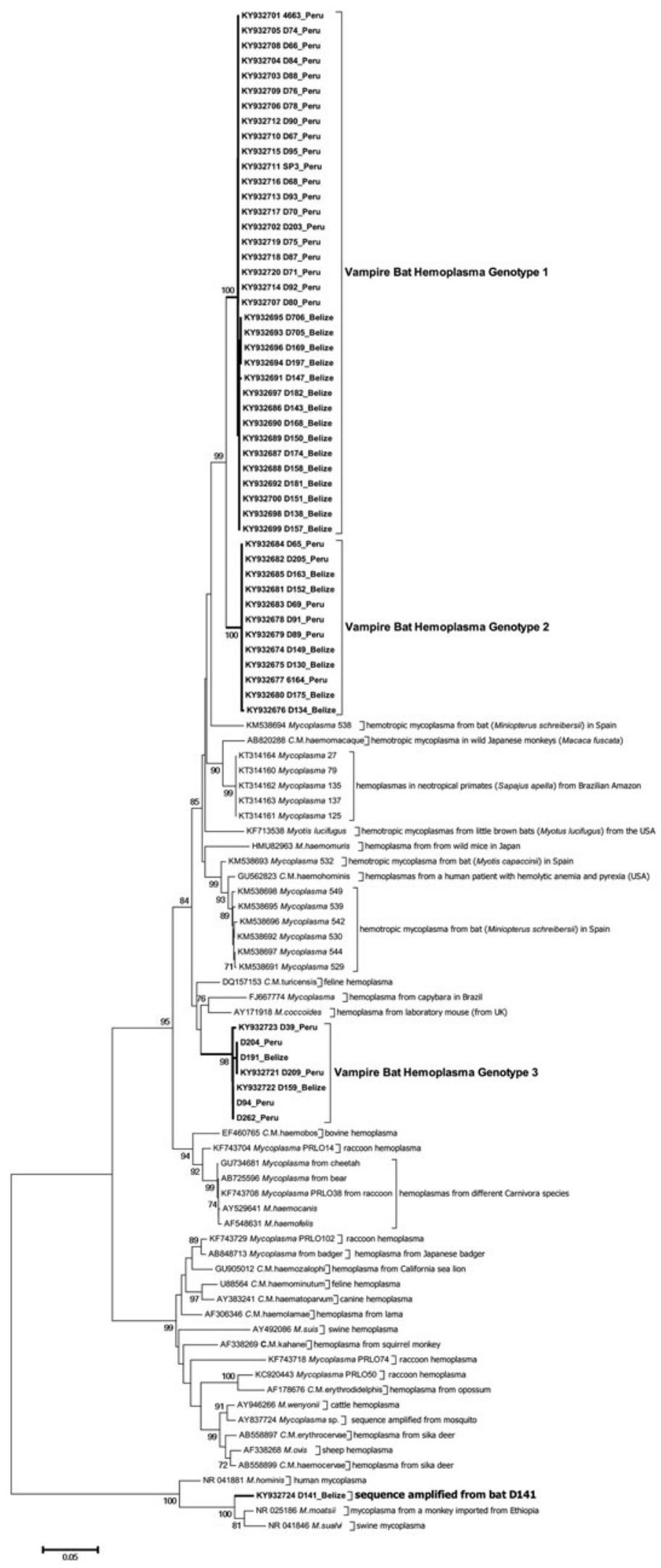

Fig. 2. Dendrogram showing phylogenetic relationships based on nucleotide sequence data for the $16 \mathrm{~S}$ rRNA gene among the hemoplasma genotypes detected in common vampire bats (Desmodus rotundus) with other hemotropic Mycoplasma spp. The tree was constructed using the minimum evolution method in MEGA 6. Accession numbers for sequences downloaded from GenBank are shown alongside individual bat ID and country of sampling. The Desmodus rotundus samples sequenced in this study are displayed in bold. 
(a)
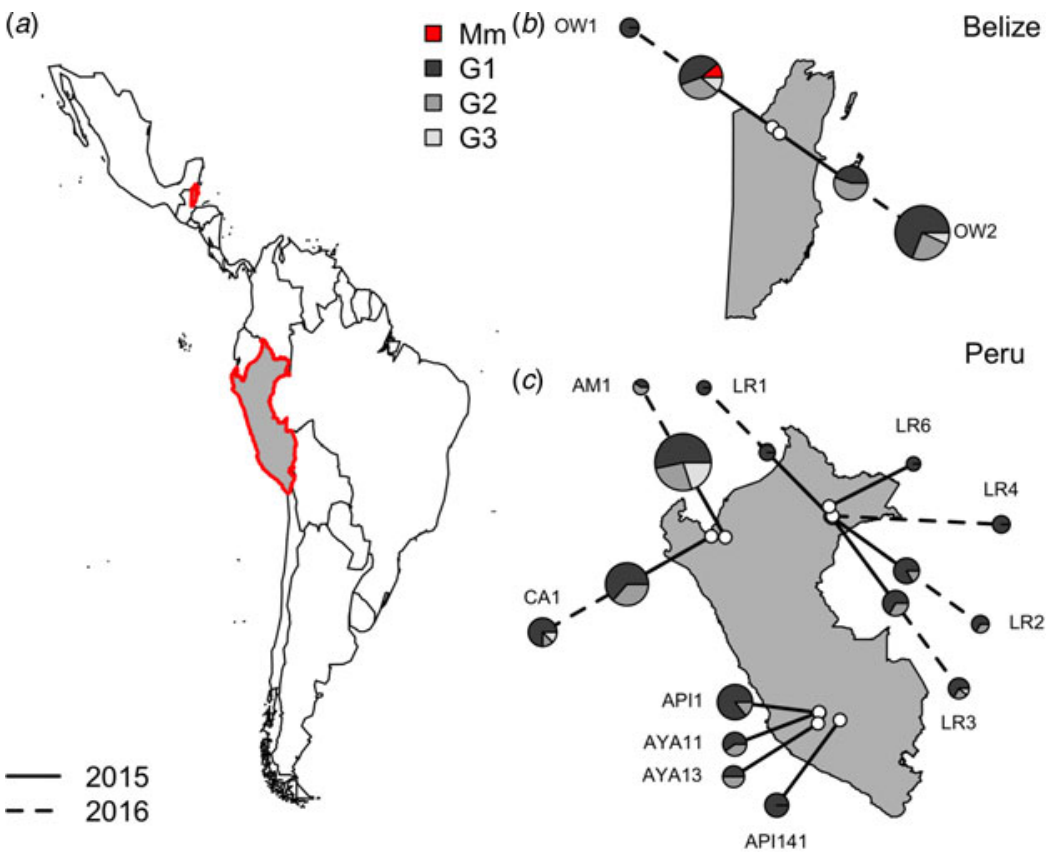

Fig. 3. Location of vampire bat sampling sites in Latin America, with Belize and Peru showed in gray with red outlines (a). Insets show the location of field sites (white) and the composition of hemoplasma genotypes per site $(b$ and $c)$ across study years (solid line $=2015$, dashed line $=2016$ ). Points are scaled by sample size. Mm denotes the M. moatsii-like hemoplasma.

than one (Fig. $4 a$ ), although the $95 \%$ confidence interval for subadults only just overlapped with one $(\mathrm{OR}=$ $1 \cdot 50,95 \% \mathrm{CI}=1 \cdot 00-2 \cdot 26$; Fig. $4 a$ ). A weaker effect was observed for males $(\mathrm{OR}=1 \cdot 21,95 \% \mathrm{CI}=0 \cdot 85$ 1.66). Hemoplasma infection prevalence was thus greatest for non-reproductive bats, especially those sampled in the fall, and for subadult bats (Fig. $4 b$, $c$ ). The odds of hemoplasma infection did not vary across the two countries and two years (Fig. 4a). In a small sample of recaptured bats $(n=6)$, we observed two bats move from infected to uninfected status within 367-371 days, while two individuals remained infected across 369-424 days (Supplementary Fig. S2).

\section{Comparison of Mycoplasma species detected in blood and saliva}

Based on the saliva metagenomic data, we identified contigs closely matching to Mycoplasma species in all oral swab sample pools from Peru (Supplementary Table S4). We consistently found long contigs (>1300 bp) with high coverage likely belonging to nonhemoplasma Mycoplasma species present in vampire bat saliva. Across all study regions, we also identified shorter contigs (400-500 bp) with high sequence identity $(98-100 \%)$ to the hemoplasma sequences of genotypes 1 and 3 that were detected in the blood of
D. rotundus. This suggests the possible presence of similar hemoplasmas in both vampire bat saliva and blood, although the shorter length of these contigs prohibited conclusive identification.

\section{DISCUSSION}

Our study describes a novel and genetically diverse hemoplasmas in common vampire bats. Infection prevalence was relatively high for this species $(67 \%)$ compared with that of from other bats, with all three novel genotypes being geographically widespread. The odds of hemoplasma infection were greatest for non-reproductive bats sampled in the fall and for subadult bats and did not vary between 2015 and 2016 both within and across countries, suggesting individuals important to transmission and endemic infection dynamics. Salivary metagenomics also showed the presence of non-hemotropic Mycoplasma species and hemoplasma genotypes phylogenetically similar to those identified in blood, providing indirect data for possible direct transmission of hemoplasmas in vampire bats through biting or grooming.

The genus Mycoplasma currently comprises 20 hemoplasma species (NIH NCBI Taxonomy). Except for M. haemocanis, M. haemofelis, and $M$. 

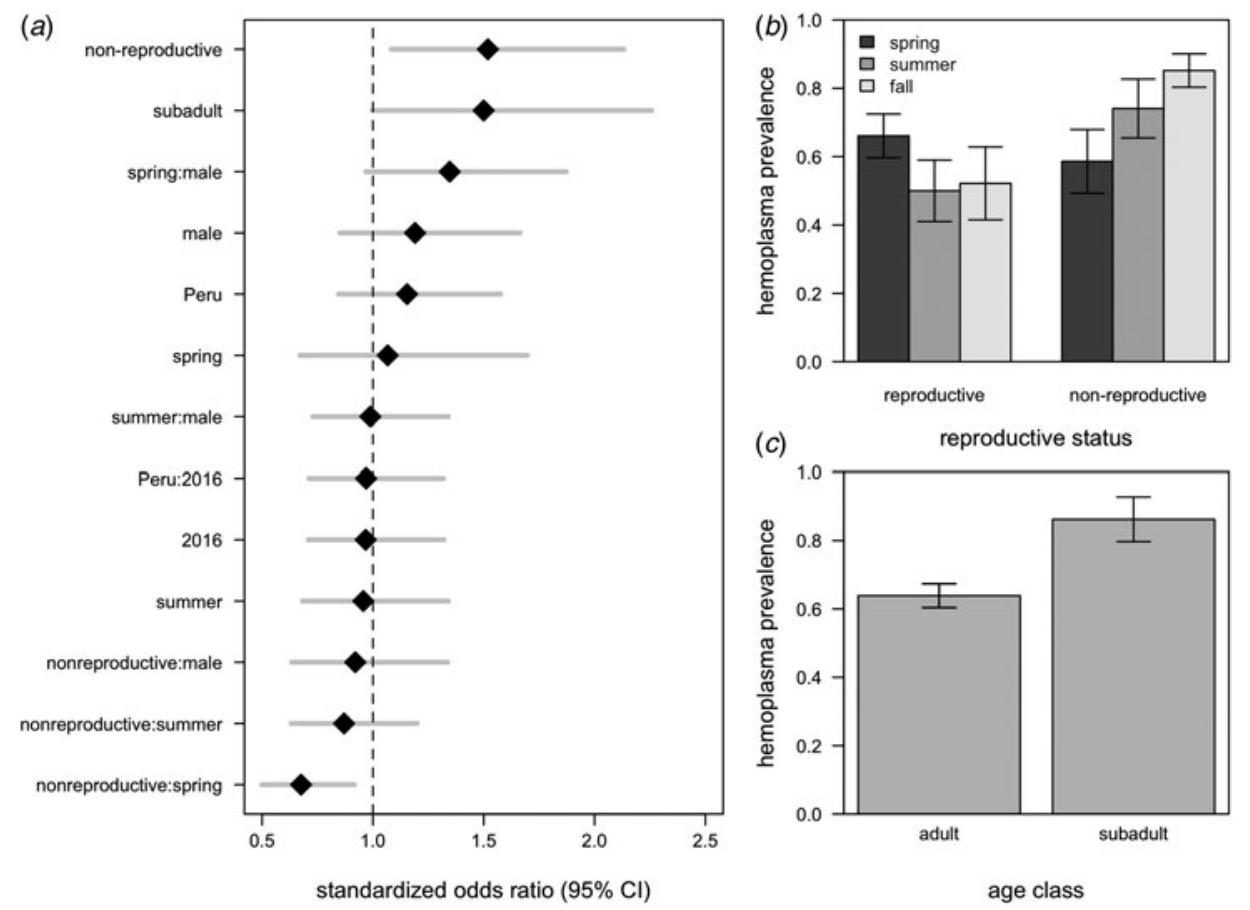

Fig. 4. Averaged odds ratios and 95\% confidence intervals for all variables within the 95\% GLMM set, standardized by partial standard deviation $(a)$. The dashed line shows where the odds ratio equals 1. Raw hemoplasma infection prevalence and $95 \%$ confidence intervals for bat reproductive status by sampling season $(b)$ and age class $(c)$.

haemomuris, hemoplasmas have the provisional taxonomic status 'Candidatus' because they are uncultivated and as a result are incompletely characterized bacterial species $[56,75,76]$. Here, PCR screening using the previously published UNI_16S_mycF and UNI_16S_mycR universal primers [50] and amplification of the full-length 16S rRNA gene using PCR primers designed in this study illustrate these primers can be used for detection of multiple hemotropic Mycoplasma spp. genotypes in vampire bats. This is also the first study in which non-hemotropic Mycoplasma species were detected in vampire bats using metagenomics. Mycoplasma species are a part of the normal oral, intestinal, and genital microflora in many animals. We know of no published references on non-hemotropic Mycoplasma species in bats, with the exception of a high presence of Mycoplasma spp. 16S rRNA gene sequences detected in intestinal biopsy samples of Cynopterus spp. bats [18]. Based on our metagenomics data, we cannot infer the significance of finding non-hemotropic Mycoplasma in bat saliva on the health of these animals or potential interspecies transmission. More research is needed on the normal Mycoplasma microflora in bats.

Phylogenetic studies of the 16S rRNA gene of closely related Mycoplasma species (including hemoplasmas) propose to use the arbitrary interspecies sequence similarity value of $\leqslant 97 \%$ as a minimum level indicating a separate, genetically distant species [76,77]. Data based on the expanded analysis of the 16S rRNA gene sequences of the species within the family Mycoplasmataceae generally support this proposition [56]. All three vampire bat hemoplasma genotypes demonstrated low levels (i.e. $<97 \%$ ) of sequence identity to previously described genotypes (or hemoplasma species) detected in other animal species, which suggests that these vampire bat hemoplasmas are novel hemoplasma genotypes or putatively new hemoplasma species not yet described in other animals $[56,57]$.

The vampire bat genotypes are paraphyletic to each other and appear to have common ancestry with hemoplasmas from other bats, rodents, humans, and non-human primates, suggesting that hemoplasmas have a history of host shifts between closely and distantly related species during evolution. Additionally, we observed no geographic clustering for genotypes 2 and 3, suggesting vampire bat hemoplasmas are broadly distributed across Latin America. However, for hemoplasma genotype 1, sequences from Belize and Peru had geography-specific single-nucleotide polymorphism (SNPs) and varied by $2 \cdot 2 \%$ (20 SNPs of $871 \mathrm{nt}$ analyzed sequence); these sequences fell into two country-specific groups (Fig. 2); this might 
imply more regionally constrained transmission cycles of this hemoplasma genotype.

Hemoplasma infection prevalence observed here in vampire bats $(67 \%)$ was intermediate compared with that in other bat species. Hemoplasma prevalence in Schreibers' bats (M. schreibersii) and one long-eared bat (M. capaccinii) in Spain was 97\% [17], while only $47 \%$ of little brown bats (M. lucifugus) from the eastern and northeastern USA and only $14 \%$ of velvety free-tailed bats $(M$. molossus) were infected with hemoplasmas $[16,19]$. However, the sensitivity of our PCR has not been quantified, so prevalence in vampire bats could conceivably be higher than we detected. Hemoplasmas have not been cultured in vitro, and their detection in many species has used PCR with or without analysis of Romanowsky-Giemsa and acridine orange-stained blood smears [20,57]. Prior work on bats has relied on PCR only but either with blood preserved in EDTA or with spleen, liver, or heart tissues $[16,17,19]$. We instead used blood preserved on Whatman FTA cards to facilitate roomtemperature sample storage in remote, tropical field conditions. During primary PCR screening, we found only $30 \%$ of positive samples produced a strong band through gel electrophoresis; other positive samples produced average or weak bands and we were mostly unable to amplify the full-length $16 \mathrm{~S}$ rRNA gene from such samples. Similar problems with amplification of hemoplasma-specific PCR products were recently identified in other bat species in Brazil [19]. Two possibilities for the high number of weak band samples in primary PCR are that hemoplasma concentrations in vampire bat blood are low or that the use of current sample collection or storage methods is inefficient for hemoplasma characterization in bats. We did not detect hemoplasmas in blood using light microscopy, though the sensitivity of this method is relatively low compared to PCR [78]. Further, manual staining in field conditions often results in stain precipitate, which makes a definitive detection of hemoplasmas through microscopy difficult.

Within vampire bats, we found the odds of hemoplasma infection to be greatest for non-reproductive bats sampled in the fall and for subadult bats. Overall higher prevalence in non-reproductive bats is surprising, given that animals often down-regulate costly immune function during reproductive events and are more susceptible to infection $[79,80]$. This pattern could possibly reflect seasonal birth pulses and the influx of immunologically naïve bats $[81,82]$, which is corroborated by the OR for non-reproductive bats being greatest in the fall, which could represent a time lag after the spring births in vampire bats [83]. Seasonal birth pulses could also explain the trend for prevalence to be greater in subadult bats, though the marginally significant averaged effect of age is likely due to controlling for other factors in the GLMMs. This trend is similar to findings on rabies virus exposure, in which younger vampire bats also showed higher seroprevalence [84]. Subadults could also experience greater exposure to hemoplasmas if vectors are more attracted to younger bats [85] or if vampire bat hemoplasmas are transmitted vertically [86]. Unlike with feline and canine hemoplasmas $[87,88]$, the odds of infection did not vary by sex, suggesting sex-biased parasitism may not occur with vampire bat hemoplasmas despite males playing a key role in the spatial dynamics of vampire bat rabies [46]. More extensive sampling of vampire bats over time, alongside infection trials, is necessary to elucidate the transmission routes of these hemoplasmas. We note that even our top GLMMs only explained up to $21 \%$ of the variation in infection status (Supplementary Table S3), which highlights the roles that coinfection with other pathogens [89], differences in host physiology [87,90], or landscape factors such as food availability [57,91] could also play in determining vampire bat susceptibility and exposure to hemoplasmas.

Hemoplasma infection prevalence did not differ between years, across countries, or generally by season. While more years of data are necessary to corroborate this result, these findings suggest hemoplasmas are endemic within vampire bat populations. Along with relatively high prevalence, this stable temporal trend corroborates other work suggesting bats to be reservoirs of hemoplasmas and potentially other bacterial infections $[9,10,17]$. The repeated sampling of a small number of recaptured bats in our study sheds further light on the infection dynamics of bat hemoplasmas. We observed two bats move from infected to uninfected within 117-123 days; this could again reflect hemoplasma DNA loads that were too low to be detected by our PCR but could also suggest vampire bats can clear hemoplasma infection. Future longitudinal sampling paired with mathematical models could help infer if vampire bats undergo cycles of latency and reactivation with hemoplasmas or obtain partial immunity from infection $[92,93]$.

To conclude, this study identified novel hemoplasma genotypes in vampire bats that were 
phylogenetically related to hemoplasmas reported in other mammals, including bats, rodents, humans, and non-human primates. These hemoplasma sequences clustered into three novel genotypes were most prevalent in young and non-reproductive bats, and were relatively stable in prevalence over time. Future studies should: (i) explore the host range and specificity of hemoplasmas among bat species and (ii) evaluate the pathogenicity of hemoplasmas in vampire bats with hematological and immunological assays. Given the close association between these vampire bat genotypes and those from humans, rodents, and non-human primates, future studies should aim to elucidate the potential for pathogen exchange between vampire bats and sympatric wildlife, humans, and domestic animals. Our metagenomic data identifying Mycoplasma species and similar hemoplasma genotypes in vampire bat saliva suggest the possibility for acquisition of hemoplasmas from reservoir hosts or for direct transmission of hemoplasmas through biting during aggressive encounters with conspecifics [94], blood sharing [95], and feeding on prey [38], but infection trials are needed to confirm this transmission route and its zoonotic potential.

\section{SUPPLEMENTARY MATERIAL}

The supplementary material for this article can be found at https://doi.org/10.1017/S095026881700231X

\section{ACKNOWLEDGEMENTS}

For assistance with site identification and field logistics, we thank Patricia Mendoza, regional offices and hospitals of the Ministry of Health in Chiclayo and Iquitos in Peru, and staff of the Wildlife Conservation Society Peru, Programa de Conservación de Murciélagos del Peru, and Lamanai Field Research Center. We also thank residents of communities along the Chiriaco, Marañon, Tahuayo, Nanay, and Yanayacu rivers in Peru for accommodations and transportation during fieldwork. For assistance with bat sampling and research permits, we thank Jorge Carrera, Pierre Castro, Miluska Ramos, Marcela Oversluijs, Cindy Quino, Carlos Tello, Nestor Falcon, Carlos Shiva, John Claxton, Ornela Inagaki, Brock Fenton, Nancy Simmons, Mark Howells, Neil Duncan, John Hermanson, Alexandra Bentz, and staff of the Instituto Nacional de Salud Peru and Lamanai Field Research Center. We thank Annie Page-Karjian, Cecilia Nachtmann, and Katherine
Smith for assistance with DNA extractions and thank Ana da Silva Filipe, Chris Davis, and Alice Broos for assistance with metagenomics labwork. We also thank anonymous reviewers for providing critical review comments on an earlier draft of this manuscript.

DJB was funded by an NSF Graduate Research Fellowship, ARCS Foundation Award, Sigma Xi, the Odum School of Ecology, the American Society of Mammalogists, the UGA Graduate School, the Explorer's Club, and a NSF Doctoral Dissertation Improvement Grant (DEB-1601052). SA acknowledges support from NSF DEB-1518611, RJO was supported by the UK Medical Research Council (MC_UU_12014/12), and DGS was supported by a Sir Henry Dale Fellowship, jointly funded by the Wellcome Trust and Royal Society (102507/Z/13/Z).

\section{DECLARATION OF INTEREST}

None.

\section{REFERENCES}

1. Hayman DTS, et al. Ecology of zoonotic infectious diseases in bats: current knowledge and future directions. Zoonoses and Public Health 2013; 60: 2-21.

2. Calisher CH, et al. Bats: important reservoir hosts of emerging viruses. Clinical Microbiology Reviews 2006; 19: $531-545$.

3. Stoner-Duncan B, Streicker DG, Tedeschi CM. Vampire bats and rabies: toward an ecological solution to a public health problem. PLoS Neglected Tropical Diseases 2014; 8: e2867.

4. Plowright RK, et al. Ecological dynamics of emerging bat virus spillover. Proceedings of the Royal Society B. The Royal Society, 2015; 282: 2014-2124.

5. Leroy EM, et al. Human Ebola outbreak resulting from direct exposure to fruit bats in Luebo, Democratic Republic of Congo, 2007. Vector-Borne and Zoonotic Diseases 2009; 9: 723-728.

6. Luis AD, et al. A comparison of bats and rodents as reservoirs of zoonotic viruses: are bats special? Proceedings of the Royal Society B: Biological Sciences 2013; 280: 2012-2753.

7. Luis AD, et al. Network analysis of host-virus communities in bats and rodents reveals determinants of cross-species transmission. Ecology Letters 2015; 18: 1153-1162.

8. Olival KJ, et al. Host and viral traits predict zoonotic spillover from mammals. Nature 2017; 546: 646-650.

9. Brook CE, Dobson AP. Bats as 'special'reservoirs for emerging zoonotic pathogens. Trends in Microbiology 2015; 23: 172-180.

10. Mühldorfer K. Bats and bacterial pathogens: a review. Zoonoses and Public Health 2013; 60: 93-103. 
11. Muhldorfer K, et al. Yersinia species isolated from bats, Germany. Emerging Infectious Diseases 2010; 16: 578-581.

12. Bunnell JE, et al. Detection of pathogenic Leptospira spp. infections among mammals captured in the Peruvian Amazon basin region. The American Journal of Tropical Medicine and Hygiene 2000; 63: 255-258.

13. Wray AK, et al. Viral diversity, prey preference, and Bartonella prevalence in Desmodus rotundus in Guatemala. EcoHealth 2016; 13:761-774.

14. Veikkolainen $\mathbf{V}$, et al. Bats as reservoir hosts of human bacterial pathogen, Bartonella mayotimonensis. Emerging Infectious Diseases 2014; 20: 960-967.

15. Lilley TM, et al. Molecular detection of Candidatus Bartonella mayotimonensis in North American Bats. Vector-Borne and Zoonotic Diseases 2017; 17: 243-246.

16. Mascarelli PE, et al. Hemotropic mycoplasmas in little brown bats (Myotis lucifugus). Parasites \& Vectors 2014; 7: 117.

17. Millán J, et al. Widespread infection with hemotropic mycoplasmas in bats in Spain, including a hemoplasma closely related to 'Candidatus Mycoplasma hemohominis'. Comparative Immunology, Microbiology and Infectious Diseases 2015; 39: 9-12.

18. Banskar S, Mourya DT, Shouche YS. Bacterial diversity indicates dietary overlap among bats of different feeding habits. Microbiological Research 2016; 182: 99-108.

19. Ikeda $\mathbf{P}$, et al. Evidence and molecular characterization of Bartonella spp. and hemoplasmas in neotropical bats in Brazil. Epidemiology \& Infection 2017; 145: 20382052.

20. Messick JB. Hemotrophic mycoplasmas (hemoplasmas): a review and new insights into pathogenic potential. Veterinary Clinical Pathology 2004; 33: 2-13.

21. Neimark H, Kocan KM. The cell wall-less rickettsia Eperythrozoon wenyonii is a Mycoplasma. FEMS Microbiology Letters 1997; 156: 287-291.

22. Neimark H, et al. Phylogenetic analysis and description of Eperythrozoon coccoides, proposal to transfer to the genus Mycoplasma as Mycoplasma coccoides comb. nov. and request for an opinion. International Journal of Systematic and Evolutionary Microbiology 2005; 55: 1385-1391.

23. Willi B, et al. From Haemobartonella to hemoplasma: molecular methods provide new insights. Veterinary Microbiology 2007; 125: 197-209.

24. Willi B, et al. Identification, molecular characterization, and experimental transmission of a new hemoplasma isolate from a cat with hemolytic anemia in Switzerland. Journal of Clinical Microbiology 2005; 43: 2581-2585.

25. Museux K, et al. In vivo transmission studies of 'Candidatus Mycoplasma turicensis' in the domestic cat. Veterinary Research 2009; 40: 1, 45-14.

26. Dean RS, et al. Use of real-time PCR to detect Mycoplasma haemofelis and 'Candidatus Mycoplasma haemominutum' in the saliva and salivary glands of haemoplasma-infected cats. Journal of Feline Medicine and Surgery 2008; 10: 413-417.

27. Groebel K, et al. Mycoplasma suis invades porcine erythrocytes. Infection and Immunity 2009; 77: 576-584.
28. Maggi RG, et al. Novel hemotropic Mycoplasma species in white-tailed deer (Odocoileus virginianus). Comparative Immunology, Microbiology and Infectious Diseases 2013; 36: 607-611.

29. Bonato L, et al. Occurrence and molecular characterization of Bartonella spp. and hemoplasmas in neotropical primates from Brazilian Amazon. Comparative Immunology, Microbiology and Infectious Diseases 2015; 42: 15-20.

30. Sykes JE, et al. Human coinfection with Bartonella henselae and Two hemotropic mycoplasma variants resembling Mycoplasma ovis. Journal of Clinical Microbiology 2010; 48: 3782-3785.

31. Pires dos Santos A, et al. Hemoplasma infection in HIVpositive patient, Brazil. Emerging Infectious Diseases 2008; 14: 1922-1924.

32. Webster D, et al. Chronic bronchitis in immunocompromised patients: association with a novel mycoplasma species. European Journal of Clinical Microbiology and Infectious Diseases 2003; 22: 530-534.

33. Bosnic D, et al. Rare zoonosis (hemotrophic mycoplasma infection) in a newly diagnosed systemic lupus erythematosus patient followed by a Nocardia asteroides pneumonia. The Brazilian Journal of Infectious Diseases 2010; 14: 92-95.

34. Yang Z, et al. Haemotrophic mycoplasma: review of aetiology and prevalence. Reviews in Medical Microbiology 2007; 18: 1-3.

35. Steer JA, et al. A novel hemotropic mycoplasma (hemoplasma) in a patient with hemolytic anemia and pyrexia. Clinical Infectious Diseases 2011; 53: e147-e151.

36. Sashida H, et al. Two clusters among Mycoplasma haemomuris strains, defined by the $16 \mathrm{~S}-23 \mathrm{~S}$ rRNA intergenic transcribed spacer sequences. Journal of Veterinary Medical Science 2013; 75: 643-648.

37. Pitcher DG, Nicholas RAJ. Mycoplasma host specificity: fact or fiction? The Veterinary Journal 2005; 170: 300-306.

38. Greenhall AM, Schmidt U. Natural History of Vampire Bats. Boca Raton, FL: CRC Press, Inc., 1988.

39. Voigt CC, Kelm DH. Host preference of the common vampire bat (Desmodus rotundus; Chiroptera) assessed by stable isotopes. Journal of Mammalogy 2006; 87: 1-6.

40. Streicker DG, Allgeier JE. Foraging choices of vampire bats in diverse landscapes: potential implications for land-use change and disease transmission. Journal of Applied Ecology 2016; 53: 1280-1288.

41. Bobrowiec PED, Lemes MR, Gribel R. Prey preference of the common vampire bat (Desmodus rotundus, Chiroptera) using molecular analysis. Journal of Mammalogy 2015; 96: 54-63.

42. Schneider MC, et al. Rabies transmitted by vampire bats to humans: an emerging zoonotic disease in Latin America?. Revista Panamericana de Salud Pública 2009; 25: 260-269.

43. Condori-Condori RE, et al. Enzootic and epizootic rabies associated with vampire bats, Peru. Emerging Infectious Diseases 2013; 19: 1463.

44. da Mendes WS, et al. An outbreak of bat-transmitted human rabies in a village in the Brazilian Amazon. Revista de Saúde Pública 2009; 43: 1075-1077. 
45. Schaer J, et al. Epauletted fruit bats display exceptionally high infections with a hepatocystis species complex in South Sudan. Scientific Reports 2017; 7: 6928.

46. Streicker DG, et al. Host-pathogen evolutionary signatures reveal dynamics and future invasions of vampire bat rabies. Proceedings of the National Academy of Sciences of the United States of America 2016; 113: 10926-10931.

47. Becker DJ, et al. Predictors and immunological correlates of sublethal mercury exposure in vampire bats. Royal Society Open Science 2017; 4: 170073.

48. Delpietro HA, Russo RG. Observations of the common vampire bat (Desmodus rotundus) and the hairy-legged vampire bat (Diphylla ecaudata) in captivity. Mammalian Biology-Zeitschrift für Säugetierkunde 2002; 67: 65-78.

49. Ahmed HA, et al. The best practice for preparation of samples from FTA $^{\circledR}$ cards for diagnosis of blood borne infections using African trypanosomes as a model system. Parasites \& Vectors 2011; 4: 1-7.

50. Volokhov DV, et al. Novel hemotrophic mycoplasma identified in naturally infected California sea lions (Zalophus californianus). Veterinary Microbiology 2011; 149: 262-268.

51. Christen R. Identifications of pathogens - a bioinformatic point of view. Current Opinion in Biotechnology 2008; 19: 266-273.

52. Ashelford KE, et al. New screening software shows that most recent large 16S rRNA gene clone libraries contain chimeras. Applied and Environmental Microbiology 2006; 72: 5734-5741.

53. Hugenholtz $\mathbf{P}$, Huber T. Chimeric $16 \mathrm{~S}$ rDNA sequences of diverse origin are accumulating in the public databases. International Journal of Systematic and Evolutionary Microbiology 2003; 53: 289-293.

54. Wright ES, Yilmaz LS, Noguera DR. DECIPHER, a search-based approach to chimera identification for 16S rRNA sequences. Applied and Environmental Microbiology 2012; 78: 717-725.

55. Edgar RC, et al. UCHIME improves sensitivity and speed of chimera detection. Bioinformatics 2011; 27: 2194-2200.

56. Volokhov DV, et al. RNA polymerase beta subunit (rpoB) gene and the 16S-23S rRNA intergenic transcribed spacer region (ITS) as complementary molecular markers in addition to the 16S rRNA gene for phylogenetic analysis and identification of the species of the family. Mycoplasmataceae. Molecular Phylogenetics and Evolution 2012; 62: 515-528.

57. Volokhov DV, et al. Prevalence, genotype richness, and coinfection patterns of hemotropic mycoplasmas in raccoons (Procyon lotor) in environmentally protected and urbanized barrier islands. Applied and Environmental Microbiology 2017; 83(9): 00211-17.

58. R Core Team. R: A Language and Environment for Statistical Computing. Vienna, Austria: R Foundation for Statistical Computing, 2013.

59. Hope ACA. A simplified Monte Carlo Significance test procedure. Journal of the Royal Statistical Society Series B (Methodological) 1968; 30: 582-598.
60. Benjamini Y, Hochberg Y. Controlling the false discovery rate: a practical and powerful approach to multiple testing. Journal of the Royal Statistical Society. Series B (Methodological) 1995; 57: 289-300.

61. Zuur A, et al. Mixed Effects Models and Extensions in Ecology with $R$. New York, NY: Springer Science \& Business Media; 2009.

62. Nakagawa S, Schielzeth H. A general and simple method for obtaining R2 from generalized linear mixed-effects models. Methods in Ecology and Evolution 2013; 4: 133-142.

63. Burnham KP, Anderson DR. Model Selection and Multimodel Inference: A Practical Information-Theoretic Approach. New York, NY: Springer Science \& Business Media, 2002.

64. Cade BS. Model averaging and muddled multimodel inferences. Ecology 2015; 96: 2370-2382.

65. Barton K. MuMIn: Multi-model inference. R package version 1.9. 5. 2013.

66. Venables WN, Ripley BD. Modern Applied Statistics with S-PLUS. New York, NY: Springer Science \& Business Media, 2013.

67. Graf EH, et al. Unbiased detection of respiratory viruses by use of RNA sequencing-based metagenomics: a systematic comparison to a commercial PCR panel. Journal of Clinical Microbiology 2016; 54: 1000-1007.

68. Yang $\mathbf{J}$, et al. Unbiased parallel detection of viral pathogens in clinical samples by use of a metagenomic approach. Journal of Clinical Microbiology 2011; 49: 3463-3469.

69. Plaire D, et al. Comparative analysis of the sensitivity of metagenomic sequencing and PCR to detect a biowarfare simulant (Bacillus atrophaeus) in soil samples. PLoS ONE 2017; 12: e0177112.

70. Martin M. Cutadapt removes adapter sequences from high-throughput sequencing reads. EMBnet Journal 2011; 17: 10-12.

71. Babraham Bioinformatics. FastQC: a quality control tool for high throughput sequence data. Cambridge, UK: Babraham Institute 2011. (https://www.bioinformatics. babraham.ac.uk/projects/fastqc/). Accessed 4 October 2017.

72. Schmieder R, Edwards R. Quality control and preprocessing of metagenomic datasets. Bioinformatics 2011; 27: 863-864.

73. Altschul SF, et al. Basic local alignment search tool. Journal of Molecular Biology 1990; 215: 403-410.

74. Bankevich A, et al. SPAdes: a new genome assembly algorithm and its applications to single-cell sequencing. Journal of Computational Biology 2012; 19: 455-477.

75. Murray RGE, Stackebrandt E. Taxonomic note: implementation of the provisional status candidatus for incompletely described procaryotes. International Journal of Systematic and Evolutionary Microbiology 1995; 45: 186-187.

76. Brown DR, Whitcomb RF, Bradbury JM. Revised minimal standards for description of new species of the class Mollicutes (division Tenericutes). International Journal of Systematic and Evolutionary Microbiology 2007; 57: 2703-2719.

77. Pettersson B, et al. Updated phylogenetic description of the Mycoplasma hominis cluster (Weisburg et al. 1989) based 
on 16S rDNA sequences. International Journal of Systematic and Evolutionary Microbiology 2000; 50: 291-301.

78. Tasker S, Lappin MR. Haemobartonella felis: recent developments in diagnosis and treatment. Journal of Feline Medicine and Surgery 2002; 4: 3-11.

79. Martin LB, Weil ZM, Nelson RJ. Seasonal changes in vertebrate immune activity: mediation by physiological trade-offs. Philosophical Transactions of the Royal Society B: Biological Sciences 2008; 363: 321-339.

80. Plowright RK, et al. Reproduction and nutritional stress are risk factors for Hendra virus infection in little red flying foxes (Pteropus scapulatus). Proceedings of the Royal Society B: Biological Sciences 2008; 275: 861-869.

81. George DB, et al. Host and viral ecology determine bat rabies seasonality and maintenance. Proceedings of the National Academy of Sciences of the Unites States of America 2011; 108: 10208-10213.

82. Amman BR, et al. Seasonal pulses of marburg virus circulation in Juvenile Rousettus aegyptiacus bats coincide with periods of increased risk of human infection. PLoS Pathogens 2012; 8: e1002877.

83. Delpietro HA, et al. Reproductive seasonality, sex ratio and philopatry in Argentina's common vampire bats. Royal Society Open Science 2017; 4: 160959.

84. Streicker DG, et al. Ecological and anthropogenic drivers of rabies exposure in vampire bats: implications for transmission and control. Proceedings of the Royal Society B: Biological Sciences 2012; 279: 3384-3392.

85. Christe, Arlettaz, Vogel. Variation in intensity of a parasitic mite (Spinturnix myoti) in relation to the reproductive cycle and immunocompetence of its bat host (Myotis myotis). Ecology Letters 2000; 3: 207-212.

86. Fujihara Y, et al. Prevalence of hemoplasma infection among cattle in the western part of Japan. Journal of Veterinary Medical Science 2011; 73: 1653-1655.
87. Walker Vergara R, et al. Prevalence, risk factor analysis, and hematological findings of hemoplasma infection in domestic cats from Valdivia, Southern Chile. Comparative Immunology, Microbiology and Infectious Diseases 2016; 46: 20-26.

88. Soto F, et al. Occurrence of canine hemotropic mycoplasmas in domestic dogs from urban and rural areas of the Valdivia Province, southern Chile. Comparative Immunology, Microbiology and Infectious Diseases 2017; 50: 70-77.

89. Willi B, et al. Worldwide occurrence of feline hemoplasma infections in wild felid species. Journal of Clinical Microbiology 2007; 45: 1159-1166.

90. Hawley DM, Altizer SM. Disease ecology meets ecological immunology: understanding the links between organismal immunity and infection dynamics in natural populations. Functional Ecology 2011; 25: 48-60.

91. Becker DJ, Streicker DG, Altizer S. Linking anthropogenic resources to wildlife-pathogen dynamics: a review and meta-analysis. Ecology Letters 2015; 18: 483-495.

92. Blackwood JC, et al. Resolving the roles of immunity, pathogenesis, and immigration for rabies persistence in vampire bats. Proceedings of the National Academy of Sciences of the United States of America 2013; 110: 20837-20842.

93. Plowright RK, et al. Transmission or within-host dynamics driving pulses of zoonotic viruses in reservoir-host populations. PLoS Neglected Tropical Diseases 2016; 10: e0004796.

94. Greenhall AM, Schmidt U, Lopez-Forment W. Attacking behavior of the vampire Bat, Desmodus rotundus, under field conditions in Mexico. Biotropica 1971; 3: 136.

95. Wilkinson GS. Reciprocal food sharing in the vampire bat. Nature 1984; 308: 181-184. 